\title{
Efecto de la raza sobre la calidad de la canal y de la carne en corderos Pampinta y su cruza con Hampshire Down
}

\section{Effect of breed on carcass and meat quality in Pampinta lambs and their crossing with Hampshire Down}

\section{Efeito da raça na carcaça e na qualidade da carne de cordeiros Pampinta e seu cruzamento em Hampshire Down}

\author{
Stazionati $M F^{1}$, Keilty $H^{2}$, Sánchez, $H^{2}$ y Murcia, $V N^{1}$ \\ 1 Estacion Experimental Agropecuaria "Ing. Agr. Guillermo Covas". Ruta Nac. 5. Anguil. La Pampa \\ 2 Facultad de Ciencias Veterinarias de la Universidad Nacional de Rosario. Ovidio Lagos 1000. Casilda. \\ Correo electrónico: stazionati.micaela@inta.gob.ar
}

\section{RESUMEN}

Los cruzamientos constituyen una importante herramienta para mejorar la producción de carne ovina. El objetivo del presente trabajo fue evaluar las características de la canal y carne de corderos Pampinta y su cruza con Hampshire Down. Para el estudio se utilizaron 23 corderos machos enteros, 11 de la raza Pampinta y 12 cruzas Pampinta con Hampshire Down. Los corderos fueron controlados sanitariamente y pesados semanalmente. Al pie de la madre pastorearon en verdeos de triticale (Triticosecale aestivum; L.) con vicia (Vicia sativa). Fueron destetados con un peso promedio de $20,2(4,22) \mathrm{kg}$ de peso vivo (PV) y una CC de 2,5 en promedio (escala de 1 a 5). Ingresaron a engorde a corral en un solo grupo durante 84 días. El día de la faena se registró el PV Pampinta 37,25 (1,76) kg; Hampshire Down $37,25(1,81) \mathrm{kg}$, peso de la canal caliente; conformación y engrasamiento; la grasa (pélvico-renal) para la categoría de más de $13 \mathrm{~kg}$ de canal; $\mathrm{pH}$ de la canal caliente; LC y LP; AG y AT e índices de compacticidad calculados a partir de las medidas morfométricas y el peso. Luego de $12 \mathrm{~h}$ de maduración se 
midió PCE, pH y se determinó en el músculo longissimus thorasis el EGD; espesor del tejido subcutáneo en el punto GR; AOB; FC con la cizalla WarnerBrätzler; CRA y PPC. Los animales se clasificaron conforme las letras S, E, U, R, O y P; según gran desarrollo muscular hasta una marcada carencia muscular, y se estipuló para la terminación 5 grados, desde la carencia de cobertura grasa hasta la excesiva presencia. No hubo efecto de la raza $(\mathrm{P}=$ 0,05 ) en todas las características de la canal. Para el análisis de los datos se utilizó el análisis de varianza (ANOVA). Estos resultados reflejan diferencias en la conformación de la canal.

Palabras claves: corderos, calidad, canal, carne, Pampinta, Hampshire Down

\section{ABSTRACT}

Crossbreeding is an important tool to improve sheep meat production. The objective of the present work was to evaluate the characteristics of the carcass and meat of Pampinta lambs and their cross with Hampshire Down. For the study, 23 whole male lambs were used, 11 of the Pampinta breed and 12 Pampinta crosses with Hampshire Down. The lambs were checked healthily and weighed weekly. At the mother's foot they grazed on triticale (Triticosecale aestivum; L.) greens with vetch (Vicia sativa). They were weaned with an average weight of 20.2 (4.22) kg live weight (LW) and a WC of 2.5 on average (scale from 1 to 5). They entered a pen in a single group for 84 days. On the day of slaughter, the PV Pampinta 37.25 (1.76) kg was recorded; Hampshire Down 37.25 (1.81) kg, hot carcass weight; shaping and fattening; the fat (pelvic-renal) for the category of more than $13 \mathrm{~kg}$ of carcass; hot runner $\mathrm{pH}$; LC and LP; AG and AT and compactness indices calculated from morphometric measurements and weight. After $12 \mathrm{~h}$ of maturation, PCE and $\mathrm{pH}$ were measured and EGD was determined in longissimus thorasis muscle; thickness of the subcutaneous tissue at the GR point; AOB; FC with the Warner-Brätzler shear; CRA and PPC. The animals were classified according to the letters S, E, $\mathrm{U}, \mathrm{R}, \mathrm{O}$ and P; according to great muscular development up to a marked muscular deficiency, and 5 degrees were stipulated for the completion, from the lack of fat coverage to the excessive presence. There was no effect of breed $(\mathrm{P}=0.05)$ on all carcass characteristics. Analysis of variance (ANOVA) was used for data analysis. These results reflect differences in the conformation of the carcass.

Key words lambs, carcass and meat quality, Pampinta, Hampshire Down. 


\section{RESUMO}

O cruzamento é uma ferramenta importante para melhorar a produção de carne ovina. 0 objetivo deste trabalho foi avaliar as características de carcaça e carne de cordeiros Pampinta e seu cruzamento com Hampshire Down. Para o estudo, foram utilizados 23 cordeiros machos inteiros, 11 da raça Pampinta e 12 cruzamentos Pampinta com Hampshire Down. Os cordeiros foram higienizados e pesados semanalmente. Aos pés da mãe, pastavam triticale (Triticosecale aestivum; L.) com ervilhaca (Vicia sativa). Foram desmamados com peso médio de 20,2 $(4,22) \mathrm{kg}$ de peso vivo (PV) e CC de 2,5 em média (escala de 1 a 5). Eles entraram em uma caneta em um único grupo por 84 dias. No dia do abate, foi registrado o PV Pampinta 37,25 $(1,76) \mathrm{kg}$; Hampshire Down $37,25(1,81) \mathrm{kg}$, peso de carcaça quente; modelagem e engorda; gordura (pélvico-renal) para a categoria de mais de $13 \mathrm{~kg}$ de carcaça; pH da câmara quente; LC e LP; AG e AT e índices de compacidade calculados a partir de medidas morfométricas e peso. Após $12 \mathrm{~h}$ de maturação, o PCE e o pH foram medidos e a EGD foi determinada no músculo longissimus thorasis; espessura do tecido subcutâneo no ponto GR; AOB; FC com a tesoura Warner-Brätzler; CRA e PPC. Os animais foram classificados de acordo com as letras S, E, U, R, O e P; de acordo com grande desenvolvimento muscular até uma deficiência muscular marcada, e 5 graus foram estipulados para a realização, desde a falta de cobertura de gordura à presença excessiva. Não houve efeito da raça $(\mathrm{P}$ $=0,05$ ) em todas as características de carcaça. A análise de variância (ANOVA) foi usada para a análise dos dados. Esses resultados refletem diferenças na conformação da carcaça.

Palavras-chave: cordeiros, qualidade, carcaça, carne, Pampinta, Hampshire Down

Fecha de recepción artículo original: 26-07-2021

Fecha de aceptación para su publicación: 20-09-2021

\section{Introducción}

Los cruzamientos son una importante herramienta para mejorar la producción de carne ovina, basada en machos de razas carniceras, brindando la posibilidad de generar distintos rendimientos y características diferentes en la res y la carne. ${ }^{(1)}$ La producción de carne de 
cordero bajo ciertos sistemas, especialmente aquellos extensivos pastoriles, presenta particularidades sensoriales que le permiten diferenciarse y ser valorada por los consumidores. ${ }^{(2,3)}$ Según Mueller (2013) (4) en Argentina los cruzamientos son poco comunes. Y cuando se menciona calidad de carne las razas escogidas son Hampshire Down (HD), Scottish Blackface, Texel, Suffolk y Poll Dorset. En el año 2006, Busetti (2006), (5) evaluaron el crecimiento y las características de la canal en animales predestete antes de la faena comparando Pampinta y sus cruzas con Ile de France y Texel. Por otro lado, Picardi $(2017)^{(6)}$ evaluaron el genotipo magrario en cruzas evaluando principalmente el crecimiento y engrasamiento. Existe interés por parte de los productores y criadores para obtener información especialmente sobre aspectos relacionados con el comportamiento productivo, respuesta a la selección y al sistema de cruzamientos orientados a evaluar las características de la canal de importancia económica. Ingresar en esquemas de cruzamientos como alternativa tecnológica, no implica necesariamente heterogeneizar las majadas y los productos generados, y mucho menos involucrarse en procesos irreversibles de deterioro o cambio de las características más productivas y deseables de las razas puras parentales ni de los sistemas productivos involucrados. Los cruzamientos, no establecen necesariamente efectos indeseables para la industria en términos de la compra de productos difíciles de estandarizar y de difícil obtención en los volúmenes que los hagan atractivos para el comercio internacional. Pero, si implica definir objetivos desde el comienzo de los procesos, y complementar las mejores características de cada raza o explotar la heterosis individual o maternal en beneficio del sistema productivo, combinando de forma racional, premeditada y equilibrada las mejores virtudes de cada una de ellas (7). Para entender mejor cómo opera esta herramienta de manejo, es útil recordar las bases del crecimiento del cordero. El peso y estado de gordura o "condición corporal" a una edad determinada, depende fundamentalmente de la ganancia diaria de peso y en menor medida del peso al nacimiento. La ganancia diaria es función de una interacción entre la nutrición del cordero y su potencial genético para crecer y engordar ${ }^{(8)}$.

El objetivo general de este estudio fue evaluar la utilización del vigor híbrido como herramienta productiva y comercial, a modo de valorizar el aporte de la variabilidad genética de las razas puras. Los objetivos específicos fueron evaluar el cruzamiento de Hampshire Down (HD) x Pampinta comparando la raza pura en el crecimiento/engorde, parámetros de conformación/composición de la canal, y características físicas y químicas de la carne. 


\section{Materiales y métodos}

El trabajo se realizó en las instalaciones de la Estación Experimental Agropecuaria (EEA) INTA ANGUIL de La Pampa, donde compararon dos tipos raciales, el Pampinta y la cruza Hampshire Down $x$ Pampinta (HD x P). Se utilizaron 11 Pampinta y 12 de la cruza HD x P.

Desde el nacimiento hasta la faena los corderos fueron pesados semanalmente. Desde el nacimiento y hasta el destete, los corderos y sus madres pastorearon en verdeos de invierno de triticale (Triticuma estivum) (1373 kg MS/ha; $18 \%$ (14-22) de proteína bruta (PB) en base a \% de materia seca (MS), calculada a partir del \% de nitrógeno; fibra estimada a través de la técnica de Fibra Detergente Neutro (FDN) en \% base MS 42,9 (37-49); Digestibilidad en \% en base a MS 69,3 (66-73) y 2,50 energía metabolizable $(2,37-2,63)$ expresada en megacalorías / kgMS) con vicia (Vicia villosa) (4770 $\mathrm{kgha}^{-1} ; 17,93 \%$ de PB; y 43,71 $\%$ de FDN). La materia seca total se estimó mediante el muestreo de $0,50 \mathrm{~m}$ de surco por unidad experimental (15 m-2), llegando a peso constante, en estufa a $60^{\circ} \mathrm{C}$. Una muestra de cada tratamiento fue procesada en laboratorio para proteína bruta (PB), fibra detergente neutro (FDN).

Los corderos fueron destetados el día 11/9/2018 con un peso promedio de $20,16^{(3,21)} \mathrm{kg}$ de peso vivo (PV), un promedio de edad de 39-41 días y una condición corporal promedio de $2,5^{\left({ }^{(9)}\right.}$, ingresando a engorde a corral durante 84 días.

Se impuso una dieta basada en rollo de alfalfa (Medicago sativa), alimento balanceado proteico comercial $(16 \% \mathrm{~PB})$ y grano de maíz entero en la última etapa (Tabla 1 y 2).

En la figura 1 se puede observar la evolución del crecimiento a lo largo del ensayo según la raza pura y la cruza.

Tabla 1: Características de las dietas suministradas, expresadas como gramos por animal y por día.

\begin{tabular}{|c|c|c|c|c|c|c|}
\hline \multirow{2}{*}{ Fuente } & \multirow{2}{*}{ Componente } & \multicolumn{3}{|c|}{ Dieta 1 adaptación } & Dieta 2 & Dieta 3 \\
\hline & & \multicolumn{3}{|c|}{ Día 1 a 21} & Día 22 a 38 & Día 39 al 84 \\
\hline \multirow[t]{2}{*}{ Proteica } & $\begin{array}{l}\text { poroto de soja } \\
\text { SD* }\end{array}$ & 20 & 50 & 100 & 140 & 140 \\
\hline & $\begin{array}{l}\text { balanceado } \\
\text { proteico }\end{array}$ & 100 & 250 & 500 & 700 & 700 \\
\hline Fibras & rollo alfalfa & 80 & 150 & 400 & 560 & 560 \\
\hline Energética & grano de maíz & - & - & - & - & 100 \\
\hline
\end{tabular}


Tabla 2: Valor nutritivo de los alimentos.

\begin{tabular}{|c|c|c|c|c|}
\hline Alimento & \%PB & $\begin{array}{c}\text { \%Fibra } \\
\text { (FDN }\end{array}$ & \%DIG. & Mcal EM \\
\hline Rollo de alfalfa & $15,9 \pm 3,2$ & $63,7 \pm 6,3$ & $54,9 \pm 4,9$ & $1,98 \pm 0,20$ \\
\hline Alimento proteico & $17,93 \pm 2,6$ & $25,45 \pm 5,04$ & $81,95 \pm 0,05$ & $2,97 \pm 0,03$ \\
\hline Grano de maíz & $8,8 \pm 1,5$ & $12,7 \pm 4,3$ & $86,4 \pm 0,9$ & $3,12 \pm 0,03$ \\
\hline
\end{tabular}

Análisis NIRS (FOSS NIR Systems 6500)

\section{Curva de crecimiento}

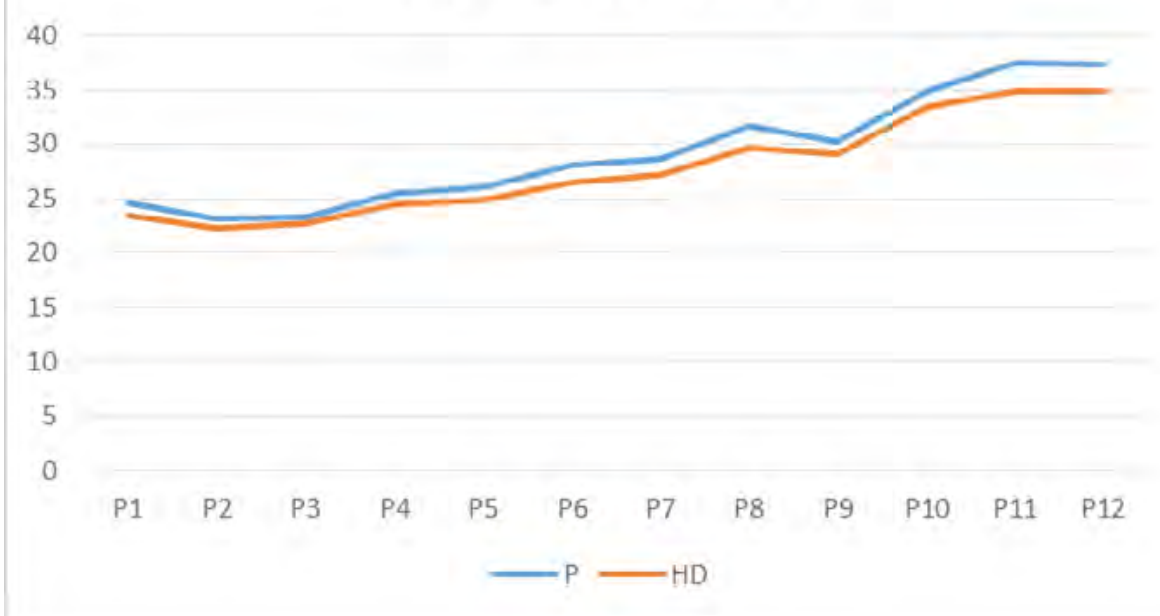

Figura 1: Evolución del crecimiento de la raza pura y las cruzas. En el eje de las abscisas están representado los pesos y en el eje de las ordenadas los días. Azul: Pampinta; Naranja: cruza P x HD.

La faena se realizó en la localidad de Anchorena (La Pampa), con un peso de $37,3(1,76) \mathrm{kg}$ en los animales cruza y $35(1,81)$ los animales puros.

A los 45 minutos post faena, se tomó el pH del músculo longissimus thorasis (LT) a la altura de la décima costilla, con electrodo Hanna instruments $囚$, modelo HI9025 para carne FC 200, USA. Luego, se determinó el peso de la canal en caliente (Rto cal). Se determinó la conformación y el nivel de engrasamiento de la canal, utilizando el estándar de clasificación de canales ovinas de la Unión Europea CEE ${ }^{(10)}$ para la categoría de más de $13 \mathrm{~kg}$ de canal. Se identificaron las distintas conformaciones con las letras S, E, U, R, O y P; según gran desarrollo muscular hasta una marcada carencia muscular, y se estipuló para la terminación 5 grados $(1,2,3,4$ y 5) desde la carencia de cobertura 
grasa hasta la excesiva presencia. La grasa pélvico-renal se midió con otra escala de notación de puntos (1-3) según Colomer-Rocher ${ }^{(11)}$; el $\mathrm{pH}$ a los 45 minutos postmortem (con electrodo Hanna instruments ${ }^{\circledR}$, modelo HI9025 para carne FC 200, USA).

Se midió en todos los animales la longitud de la canal (LC) y pierna (LP); ancho de grupa (AG) y tórax (AT) e índices de compacidad 1 (AG /LC); 2 (PCE/LC) y 3 (AG/LP) se midieron con cinta métrica, compás de reses y se calcularon los cocientes. Luego de 12 horas de maduración en cámara fría a $4 \stackrel{\circ}{\circ} \mathrm{C}$ se midió el pH final, peso de la canal enfriada (PCE) y se calcularon las mermas por frío. Posteriormente, se procedió a extraer un bloque de bife con hueso del lado derecho de cada canal, incluyendo la sección del LT en un corte transversal a la columna vertebral entre la sexta y treceava costilla. Los bloques se identificaron individualmente y fueron conservados a $4 \pm 1 \stackrel{\circ}{ } \mathrm{C}$ para la determinación de espesor de grasa dorsal (EGD) a $4 \mathrm{~cm}$ de la línea media entre la doce y treceava costilla, punto $\mathrm{GR}^{(12)}$ Área de ojo de bife del LT (AOB) por planimetría, expresado en $\mathrm{cm}^{2}$. Luego se envasaron al vacío utilizando bolsas para vacío tipo Sealed air Cryovac de $50 \mu \mathrm{m}$ y se congelaron $\mathrm{a}-20^{\circ} \mathrm{C}$,

Se realizó la cocción de dos bifes del músculo longissimus thorasis (desde la altura de la costilla T6 hasta la T12), previa descongelación a temperatura ambiente. Se cocinaron en una parrilla eléctrica (MIR 2100W grande, Parrimir, Bs. As. Argentina) hasta una temperatura interna final de $71^{\circ} \mathrm{C}$ registrada con termómetro (tipo T con vástago) insertas en el centro geométrico de cada bife. Inmediatamente después los bifes se enfriaron durante 30 minutos a temperatura ambiente y se mantuvieron a $4 \pm 1^{\circ} \mathrm{C}$ en una cámara, cubiertos con un film para evitar la desecación hasta el momento de realizar las determinaciones de fuerza de corte (FC) (las muestras se pesaron antes y después de la cocción). Posteriormente, se extrajeron de cada bife 6 cilindros del músculo longissimus thorasis con un sacabocados de $10 \mathrm{~mm}$ de diámetro, de forma paralela a la orientación de las fibras musculares. Cada cilindro fue seccionado en su parte media, perpendicular a la orientación de las fibras musculares utilizando la cizalla de corte Warner-Bratzler (Manhattan, KS, USA). Los 6 valores individuales de la FC correspondientes a cada bife se promediaron de manera de obtener un único valor por muestra, registrándose la fuerza de corte en Newton (N); los valores de pérdidas por cocción (PPC) se obtuvieron por diferencia de peso antes y después de la cocción de los bifes para la determinación de resistencia al corte, utilizando una balanza Exacta II (Máx $15 \mathrm{~kg}$, d = 1 g) con indicador Ohaus T31P. Dichos valores se expresaron en porcentaje de peso fresco. La evaluación de la capacidad 
de retención de agua (CRA), se midió a través del método de pérdidas por goteo en carne entera cruda, según la metodología propuesta por Honikel (1998) ${ }^{(13)}$

El análisis estadístico, para todas las variables, se realizó en base a un diseño totalmente aleatorizado, con el animal como unidad experimental y la raza como efecto fijo. Se utilizó el procedimiento PROC GLM SAS ${ }^{(14)}$. Cuando el efecto de un factor sobre la variable respuesta fue significativo $(\mathrm{P}<0,05)$, se utilizó la prueba de $\mathrm{F}$ de Anova.

\section{Resultados y discusión}

Respecto del peso al nacimiento, en la Tabla 3 se observa que los animales cruzas fueron más pesados, aunque no significativamente. $\mathrm{Al}$ igual que lo demostrado por Bianchi $(2005)^{(15)}$ donde no tuvo diferencias significativas en cuanto al peso de nacimiento en corderos pesados Corriedale puros y sus cruzas con HD. El efecto de la raza sobre la ganancia de peso no fue significativo, a diferencia de lo demostrado por Álvarez (2013) ${ }^{(16)}$ donde atribuye a las cruzas mayor ganancia de peso a los 90 días. A los 122 días de edad en promedio, no se encontraron diferencias significativas entre el peso y las razas. Así mismo, Busetti (2006) ${ }^{(5)}$ no obtuvo ventajas consistentes de los cruzamientos con ambas razas (Ile de France y Texel) sobre los corderos Pampinta puros. Pero si se vio afectado el peso al nacimiento por los cruzamientos, ya que los corderos puros Pampinta resultaron más pesados (7\%) que los provenientes de la cruza con Ile de France. Diaz (2001) ${ }^{(17)}$ no encontró diferencias significativas en el peso al nacimiento en corderos de madres lecheras Manchegas. Por otro lado, Dawson (2003) (18) encontró diferencias significativas en la heterosis de la ganancia diaria de peso desde el nacimiento hasta el sacrificio en el cruzamiento de las razas Rouge y Texel. Bianchi $(2003)^{(19)}$ demuestra un efecto significativo en la velocidad de crecimiento y el peso vivo en los cruzamientos de corderos Corriedale con Texel (TX), Hampshire Down (HD), Southdown (SD), Île de France (IF), Milchschaf (MI) o Suffolk (SF).

Tabla 3: Medidas de resumen de los pesos al nacimiento.

\begin{tabular}{|c|c|c|c|}
\hline Raza & N & Media & E.E \\
\hline Cruzas & 55 & 5,77 & 0,52 \\
\hline Puros & 32 & 5,62 & 0,33 \\
\hline
\end{tabular}


En el peso al destete, no hubo diferencias significativas entre los corderos puros y los corderos cruzas, siendo $23,25 \pm 1,18$ en los Pampinta y 24,58 $\pm 1,18$ en los HD x P. A diferencia de lo demostrado por Bores Quinteros (2002) ${ }^{(20)}$ donde evaluó cruzamientos terminales con padres carniceros HD y madres de pelo Pelibuey y Blackbelly, donde los cruzas HD tuvieron mayor peso al destete.

En el PV pre faena no hubo diferencias significativas $(37,25 \pm 1,81)$. Al igual Bores Quinteros $(2002)^{(20)}$ en cruzas con HD. Por otro lado, Bianchi (15) encontraron diferencias significativas en el peso al sacrificio en corderos pesados puros Corriedale y su cruza Corriedale x HD. Las medidas lineales de la canal se presentan en la tabla 4. Las medidas de longitud y anchura de la canal sirven para objetivizar y justificar su valor (21). Como ocurre con las medidas de longitud, las medidas de anchura están correlacionadas entre sí, ${ }^{(22)}$ bastando para caracterizar una canal una medida de longitud y otra de anchura. Boccard (1964) ${ }^{(22)}$ observaron que a medida que aumentaba el peso de la canal, lo hacían diversas medidas de anchura y longitud, de manera que la variación de la mayoría de estas medidas puede explicarse por la variación en el peso de la canal. En nuestro ensayo fue proporcional igual que al aumentar el peso aumentaron las medidas. Esto también lo observó Peña (2005) ${ }^{(23)}$. El crecimiento de un animal, en períodos cortos, se va a manifestar más por el aumento de su grosor (aumento de músculo y grasa), que, por el alargamiento del soporte óseo, por lo que el peso estaría más relacionado con la anchura que con la longitud. A partir de estas medidas se crearon una serie de índices, que permiten una mejor caracterización de la canal y además disminuir el efecto del peso de la canal, de gran influencia, como ya se ha dicho, sobre las mismas:(24) - Índice de compacidad de la pierna. Es el cociente entre la anchura de grupa y la longitud de la pierna (G/F) Palsson (1939) (25) - Índice de compacidad de la canal. Es el cociente entre el peso de la canal enfriada y la longitud interna de la canal (PCE/L).

Tabla 4: Efectos de las razas sobre las medidas lineales (Promedios \pm Desvío Estándar).

\begin{tabular}{|c|c|c|}
\hline \multirow{2}{*}{$\begin{array}{c}\text { Medidas } \\
(\mathrm{cm})\end{array}$} & Pampinta & Cruzas \\
\cline { 2 - 3 } & $75,91 \pm 0,98$ & $75,71 \pm 0,93$ \\
\hline LC & $21,50 \pm 0,24$ & $21,67 \pm 0,23$ \\
\hline LP & $20,86 \pm 0,48$ & $21,21 \pm 0,46$ \\
\hline AT & $22,41 \pm 0,61$ & $23,92 \pm 0,59$ \\
\hline AG &
\end{tabular}

LC: longitud de la canal; LP: longitud de la pierna; AT: ancho de tórax; AG: ancho de grupa 
No se registraron efectos $(\mathrm{P}>0,05)$ entre ambos tipos genéticos en ninguna de las medidas lineales realizadas, si bien varios autores han reportado diferencias comparando animales con diferentes pesos al sacrificio $(17,18,26,23,27)$. El mayor peso de la canal lo presentaron los animales cruzas, y en igualdad del largo de la canal $(75,91 \mathrm{P}$ vs $75,71 \mathrm{P} \mathrm{x}$ HD). Aun así, cuando la raza Pampinta siempre fue seleccionada fenotípicamente hacia un carácter lechero, donde el largo de canal es mayor que en animales de características carniceras. Si bien no se encontraron diferencias significativas para las medidas lineales en nuestro ensayo, Bianchi $(2001)^{(28)}$ propone que la utilización de razas especializadas en la producción de carne permitiría la obtención de canales pesadas, con mayor proporción de carne comestible.

Con relación a la conformación de las canales, el 77\% fueron buenas (R), a pesar de las diferencias a favor de las canales provenientes de los corderos cruzas. Datos similares fueron aportados por Bianchi $(2005)^{(15)}$ en las cruzas Corriedale x HD.

Con respecto al estado corporal, se observó una superioridad en el grado de terminación de los corderos cruzados frente a la raza pura, a pesar de que el estado corporal de los Pampinta puros fue bueno (3 vs. 2,5).

El tipo racial no afectó significativamente el peso de la canal (caliente y enfriada) Tabla 5. Datos similares fueron encontrados por Bores Quinteros (2002) ${ }^{(20)}$ en cruzamientos con HD. A diferencia de lo encontrado por Bianchi (2005) ${ }^{(15)}$ donde el tipo genético afectó significativamente los pesos de las canales tanto calientes como enfriadas.

Tabla 5: Medias y desvío estándar de los pesos de la canal caliente y enfriada.

\begin{tabular}{|c|c|c|}
\cline { 2 - 3 } \multicolumn{1}{c|}{} & Cruza & Puro \\
\hline Peso canal caliente kg & $16,93 \pm 3,71$ & $16,11 \pm 2,89$ \\
\hline Peso canal enfrida kg & $16,88 \pm 3,66$ & $16,01 \pm 2,83$ \\
\hline
\end{tabular}

Con relación a los componentes de grasa, especialmente al EGD, los animales cruzas presentaron mayores valores, pero no significativamente. Bores Quinteros (2002) ${ }^{(20)}$ observó que la raza paterna no modifica el factor graso. Busetti (2006) ${ }^{(6)}$ encontró mayores valores para la cruza Pampinta x Ille de France que los puros P. En cuanto a la clasificación de engrasamiento pélvico renal fueron pobres para ambos tanto puros como cruzas $(1,54 \pm 0,49$ vs $1,66 \pm 0,44)$.

En cuanto a las características de la carne, tanto para $\mathrm{pH}$, fuerza de corte WB y CRA no hubo diferencias significativas (Tabla 6). Los valores de fuerza de corte WB estandarizados por la industria cárnica, 
tanto de Estados Unidos como de Nueva Zelanda, para retener o acceder a nuevos mercados, deben ser menores o iguales a una fuerza de corte de $5 \mathrm{~kg}$. ${ }^{(29)}$ Los valores que se encontraron en el presente experimento se consideran satisfactorios, siendo mucho menores a los mencionados por Bianchi. ${ }^{(15,19,30,27)}$

Tabla 6: Parámetros de calidad de carne.

\begin{tabular}{|c|c|c|}
\cline { 2 - 3 } \multicolumn{1}{c|}{} & Cruza & Puros \\
\hline CRA\% & $93,05 \pm 0,66$ & $93,62 \pm 0,69$ \\
\hline AOB $(\mathrm{cm} 2)$ & $14,55 \pm 1,19$ & $13,50 \pm 1,19$ \\
\hline PCC\% & $28,26 \pm 1,71$ & $25,52 \pm 1,78$ \\
\hline Fuerza de corte $(\mathrm{Kg})$ & $0,29 \pm 0,02$ & $0,32 \pm 0,02$ \\
\hline Pto GR & $9,16 \pm 2,55$ & $5,68 \pm 1,84$ \\
\hline EGD cm & $0,17 \pm 0,02$ & $0,11 \pm 0,02$ \\
\hline
\end{tabular}

CRA: capacidad de retención de agua; AOB: área ojo de bife; PCC: perdidas por cocción; EGD: espesor de grasa dorsal.

En cuanto al AOB, fueron de mayor superficie para las cruzas, pero no se encontraron diferencias significativas. Al igual que Busetti $(2006)^{(5)}$ en las respectivas cruzas con P. No obstante Bianchi (2005) ${ }^{(15)}$ encontró diferencias significativas en los corderos pesados de cruzas con HD.

También se evaluó la PCC y el punto GR en cada uno de los animales, donde no se encontraron diferencias (Tabla 6).

\section{Conclusión}

Con los resultados del presente trabajo, podemos concluir que los corderos terminales de la raza paterna HD obtienen un crecimiento y conformación corporal similar. No se observaron mayores ventajas para todas las características evaluadas, en concordancia a lo observado por Busetti (2006) ${ }^{(5)}$ estos cruzamientos permiten la posibilidad de incorporarlos en el futuro para mejorar la conformación y el engrasamiento sin perjudicar las características de Pampinta. 


\section{Bibliografía}

1. Bianchi G, Garibotto G. Identificación y cuantificación de factores que afectan la calidad de carne ovina. En: 1er Seminario Técnico. Calidad de Carne Ovina y Vacuna: Impacto de decisiones tomadas en distintos segmentos dela cadena. (ed.) Facultad de Agronomía, EEMAC. FRICASA. Paysandú, Uruguay. Pp 52.2004.

2. Briggiler VCR. Efecto de la alimentación sobre la calidad sensorial y los compuestos aromáticos en carne de cordero y de vacuno (Doctoral dissertation, Universidad de Zaragoza). 2010.

3. Panea B, Joy M, Sanz A, Carrasco S, Delfa R. Calidad Sensorial de la carne de corderos procedentes de diferentes tipos comerciales. In: Zaragoza, C. (Ed.), Agricultura Ecológica: Gestión sostenible del agua y calidad agroalimentaria. SEAE, p.149. 2006.

4. Mueller JP. El mejoramiento genético de ovinos en Argentina. In Primer Congreso Panamericano de Ovinocultura, Querétaro, México. 2013.

5. Busetti MR, Babinec F, Suárez VH, Bedotti DO. Peso al nacimiento y crecimiento hasta el destete de corderos Pampinta y sus cruzas con Ile de France y Texel. RIA. Revista de Investigaciones Agropecuarias, 35(2), 91-101. 2006.

6. Picardi L, Keilty H, Maiztegui L, Amelong J, Acebal M, Piga E. Evaluación del genotipo magrario como progenitor en cruzamientos con otras razas ovinas. Revista Argentina de Producción Animal, 37(1), 33-39. 2017.

7. Ganzábal A, Montossi F, Ciappesoni G, Banchero G, Ravagnolo O, San Julián R et al., Cruzamientos para la producción de carne ovina de calidad: Resultados: comportamiento reproductivo y habilidad materna de ovejas, crecimiento y calidad de canal de corderos. Serie Técnica № 170. ISBN: 978-9974-38-244-2. 2007.

8. Claro MD. El cruzamiento terminal en la producción de carne ovina [en línea]. Tierra Adentro. no. 67. Disponible en: https://biblioteca.inia.cl/handle/123456789/6242 (Consultado: 15 julio 2021). 2006.

9. Russel A. Body condition scoring of sheep. In Practice 6: 91-93. 1984.

10. Reglamento delegado 2017/1182. Disponible en: https://www.colveza.es/images/ stories/normativa/DOCE/bov/RD-2017-1182clasificacion-de-canales.

11. Colomer-Rocher F, Delfa R, Sierra I. Método normalizado para el estudio de los caracteres cuantitativos y cualitativos de las canales ovinas producidas en el área mediterránea según los sistemas de producción. Cuadernos INIA 17, 19-41. 1988.

12. Kirton HA, Johnson DL. Interrelation-ship between GR and other lamb carcass fatness measurements. Proceeding of the New Zealand Society f Animal Production 39: 194201. 1979.

13. Honikel KO. Reference Methods for the Assessment of Physical Characteristics of Meat. Meat Science, Vol. 49, No. 4, 447457, 1998.

14. SAS, Statistical Analysis System User Guide Statistics. SAS Institute Inc. Cary NC 27513 USA. 1999.

15. Bianchi G. Características productivas, tipificación de la canal y calidad de carne a lo largo de la maduración de corderos pesados Corriedale puros y cruzados en sistemas 
extensivos. Tesis Doctoral. Universidad de Zaragoza, Facultad de Veterinaria, Zaragoza, España. Pp 102. 2005.

16. Álvarez JM, Mayo A, García Vinent JC, Roa M, Giorgetti H, Rodríguez G. Avances en calidad de carne de ovinos, caprinos, porcinos y aves. Avances en bienestar animal. Implicancias de la alimentación, la genética y el manejo. Publicación técnica no 93. ISSN 0325-2132/ JULIO 2013. Ediciones INTA. 2013.

17. Díaz MT. Características de la canal y de la carne de corderos lechales manchegos. Correlaciones y ecuaciones de predicción. Memoria Doctor en Med. Veterinaria. Madrid, España. U. Complutense de Madrid. Fac. de Veterinaria. 308p. 2001.

18. Dawson L, Carson A, McClinton L, Kilpatrick D, Moss B. Comparison of the carcass characteristics and meat quality of lambs produced from Texel and Rouge de l'Ouest ewes and their crosses. Animal Sci. 77:53-65. 2003.

19. Bianchi G, Garibotto G, Bentancur O. Características de crecimiento de corderos ligeros hijos de ovejas Corriedale y moruecos Corriedale, Texel, Hampshire Down, Southdown, Ile de France, Milchschaf o Suffolk. Archivos de Zootecnia, 52(199), 339-345. 2003.

20. Bores Quintero RF, Velázquez Madrazo PA, Heredia M, Aguilar M. Evaluación de razas terminales en esquemas de cruza comercial con ovejas de pelo F1 Técnica Pecuaria en México, vol. 40, núm. 1, enero-abril, pp. 71-79. 2002.

21. Aparicio F, García Arroyo L, Vera A, Vega A. Estudio de las canales de corderos Manchegos y cruzados Suffolk y Frison x Manchego. Arch. Zootec., 27, 41-50. 1978.

22. Boccard R, Dumont BL, Peyron C. Étude de la production de la viande chez les ovins. VIII. Relations entre les dimensions de la carcasse d'agneau. Ann. Zootech, 13, $367-$ 378. 1964.

23. Peña F, Cano T, Domenech V, Alcalde M, Martos J, Garcia-Martinez A, Herrera M, Rodero E. Influence of sex, slaughter weight and carcass weight on "non-carcass" and carcass quality in Segureña lambs. Small Rumin. Res. 60: 247-254. 2005.

24. Clarke EA, McMeekan CP. New Zealand lamb and mutton. N. Z. J. Sci. Technol. Agr, 33, 1-15. 1952.

25. Palsson H. Meat qualities in the sheep with special reference to Scottish breeds and crosses. Part. 1. J. Agric. Sci. Camb., 29, 544-626. 1939.

26. Revilla I, García-Martín M, Vivar-Quintana A. Efecto del peso y edad sobre las características de engrasamiento y conformación de canales de lechazo para distintas razas. XI Jornadas de producción animal AIDA. <http://www.aida-itea.org> [consulta: 1507-2021]. 2005.

27. Santos V, Silva S, Mena E, Azevedo J. Live weight and sex effects on carcass and meat quality of "Borrego Terrincho-PDO" suckling lambs. [En línea]. Meat Sci., doi: 10.1016/j.meatsci.2007.05.019. <http://www.sciencedirect.com/science> [consulta: 15-07-2021]. 2007.

28. Bianchi G. Use of breed and crossbreeding for sheep meat production in Uruguay. In: International Course of health and meat production. Conferencia. Universidad 
Austral de Chile. Facultad de Ciencias Veterinarias. Escuela de Graduados. Valdivia 17 y 18 de mayo 2001. Chile. pp. 53-69. 2001.

29. Bickerstaffe R. Proteases and meat quality. The Proceedings of the New Zealand Society of Animal Production. 56:153-162. 1996.

30. Montossi F, San Julian R, Brito G, De Los Campos G, Ganzabal A, Dighiero A, et al. Producción de carne ovina de calidad con la raza Corriedale: Recientes avances y desafíos de la innovación tecnológica en el contexto de la cadena cárnica ovina del Uruguay. In: $12^{\circ}$ Congreso Mundial de la raza Corriedale. 1-10/9/2003. Montevideo. Uruguay. Conferencia (CD-ROOM). 2003. 